\title{
Use of electroporation and reverse iontophoresis for extraction of transdermal multibiomarkers
}

\author{
Congo Tak-Shing Ching ${ }^{1,2}$ \\ Lin-Shien $\mathrm{Fu}^{3-5}$ \\ Tai-Ping Sun' \\ Tzu-Hsiang Hsu' \\ Kang-Ming Chang ${ }^{2}$
}

'Department of Electrical Engineering, National Chi Nan University, Puli, Nantou County, ${ }^{2}$ Department of Photonics and Communication Engineering, Asia University, Wufeng, Taichung, ${ }^{3}$ Department of Pediatrics, National Yang Ming University, Taipei, ${ }^{4}$ Institute of Technology, National Chi Nan University, Puli, ${ }^{5}$ Department of Pediatrics, Taichung Veterans General Hospital, Taichung City, Taiwan
Correspondence: Congo Tak-Shing Ching Department of Electrical Engineering, National Chi Nan University, I University Rd, Puli, Nantou County 5456I, Taiwan

Tel +886 4929 I 0960 ext 4774

Fax +886 4 929I 2434

Email tsching@ncnu.edu.tw

Kang-Ming Chang

Department of Photonics and

Communication Engineering, Asia

University, 500 Lioufeng Rd,Wufeng,

Taichung 41354, Taiwan

Tel +886423323456 ext 20003

Email changkm@asia.edu.tw
This article was published in the following Dove Press journal:

International Journal of Nanomedicine

22 February 2012

Number of times this article has been viewed

Background: Monitoring of biomarkers, like urea, prostate-specific antigen (PSA), and osteopontin, is very important because they are related to kidney disease, prostate cancer, and ovarian cancer, respectively. It is well known that reverse iontophoresis can enhance transdermal extraction of small molecules, and even large molecules if reverse iontophoresis is used together with electroporation. Electroporation is the use of a high-voltage electrical pulse to create nanochannels within the stratum corneum, temporarily and reversibly. Reverse iontophoresis is the use of a small current to facilitate both charged and uncharged molecule transportation across the skin. The objectives of this in vitro study were to determine whether PSA and osteopontin are extractable transdermally and noninvasively and whether urea, PSA, and osteopontin can be extracted simultaneously by electroporation and reverse iontophoresis.

Methods: All in vitro experiments were conducted using a diffusion cell assembled with the stratum corneum of porcine skin. Three different symmetrical biphasic direct currents (SBdc), five various electroporations, and a combination of the two techniques were applied to the diffusion cell via $\mathrm{Ag} / \mathrm{AgCl}$ electrodes. The three different SBdc had the same current density of $0.3 \mathrm{~mA} / \mathrm{cm}^{2}$, but different phase durations of 0 (ie, no current, control group), 30, and 180 seconds. The five different electroporations had the same pulse width of $1 \mathrm{msec}$ and number of pulses per second of 10, but different electric field strengths of 0 (ie, no voltage, control group), 74, 148, 296, and $592 \mathrm{~V} / \mathrm{cm}$. Before and after each extraction experiment, skin impedance was measured at $20 \mathrm{~Hz}$.

Results: It was found that urea could be extracted transdermally using reverse iontophoresis alone, and further enhancement of extraction could be achieved by combined use of electroporation and reverse iontophoresis. Conversely, PSA and osteopontin were found to be extracted transdermally only by use of reverse iontophoresis and electroporation with a high electrical field strength $(>296 \mathrm{~V} / \mathrm{cm})$. After application of reverse iontophoresis, electroporation, or a combination of the two techniques, a reduction in skin impedance was observed.

Conclusion: Simultaneous transdermal extraction of urea, PSA, and osteopontin is possible only for the condition of applying reverse iontophoresis in conjunction with high electroporation.

Keywords: electroporation, reverse iontophoresis, nanochannels, noninvasive, urea, prostatespecific antigen, osteopontin

\section{Background}

Prostate cancer, ${ }^{1}$ ovarian cancer, and kidney disease are global health problems. Prostate cancer holds the seventh position of leading causes of cancer deaths in Taiwan. ${ }^{2,3}$ About $50 \%$ of prostate cancer is at an advanced stage by the time the disease is detected. In Taiwan, ovarian cancer holds the second position among gynecological reproductive cancers, just behind cervical cancer. ${ }^{4}$ Kidney disease held the eighth position of leading causes of death in Taiwan in $2008,{ }^{4}$ and the prevalence of dialysis in Taiwan 
holds first place worldwide. Obviously, these statistics were the motivation for this study.

The biomarkers for prostate cancer, ovarian cancer, and kidney disease are prostate-specific antigen (PSA), ${ }^{5}$ osteopontin, ${ }^{6,7}$ and urea, respectively. A normal range for blood PSA level is generally $0.3-2.5 \mathrm{ng} / \mathrm{dL}$ for men under 50 years of age, while men (under 50 years) with prostate cancer usually have a blood PSA level ranging from 0.4 to $163 \mathrm{ng} / \mathrm{dL} .{ }^{8}$ In contrast, a normal blood osteopontin level for women is about $178 \mathrm{ng} / \mathrm{mL}$. However, women with ovarian cancer usually have a blood osteopontin level of $486.5 \mathrm{ng} / \mathrm{mL} .{ }^{9}$ Also, the normal range for blood urea in adults is generally 10-20 mg/dL. Regular checking of these biomarkers can reduce the incidence and mortality rate for people who are at high risk of these diseases. However, current clinical methods to monitor these biomarkers involve blood sampling, the pain and inconvenience of which provides considerable impetus for the development of noninvasive methods for frequent monitoring of blood PSA, osteopontin, and urea.

Skin provides a unique gateway for noninvasive monitoring. Recently, reverse iontophoresis techniques have been used for patient monitoring ${ }^{10,11}$ and noninvasive diagnosis. ${ }^{12,13}$ Electroporation techniques have also been used for noninvasive metabolite extraction, ${ }^{14}$ achieved by formation of transient nanometer-wide aqueous pores across the skin. Because of aqueous pore formation, it would be expected that electroporation can assist reverse iontophoresis for transdermal extraction of molecules with bigger size and in a shorter time.

Reverse iontophoresis refers to the passage of a low level of current through the skin to promote extraction of both charged and neutral molecules. ${ }^{15}$ However, transdermal extraction enhanced by reverse iontophoresis is limited to molecules having a molecular weight of $20 \mathrm{kDa}$ or less. ${ }^{16}$ Therefore, it can be expected that urea (molecular weight $60 \mathrm{Da}$ ) can be extracted by reverse iontophoresis. However, the molecular weights of PSA (34 kDa) and osteopontin (33 kDa) are much larger than $20 \mathrm{kDa}$, so reverse iontophoresis alone may not be able to facilitate transdermal extraction. Electroporation involves the creation of transient nanometer-wide aqueous pores across lipid bilayer membranes by applying a short, high voltage pulse. ${ }^{17}$ Research has suggested that electroporation might enhance skin permeability of molecules with a molecular weight of at least $40 \mathrm{kDa} .^{18,19}$ Therefore, combined use of reverse iontophoresis and electroporation for transdermal extraction of PSA, osteopontin, and urea may be possible.

The aims of this study were to determine whether PSA and osteopontin are extractable transdermally and noninvasively, and whether urea, PSA, and osteopontin can be extracted simultaneously by electroporation and reverse iontophoresis.

\section{Materials and methods Reagents and solutions}

All reagents used in this study are commercially available and were used without further purification. Phosphate-buffered saline and urea (molecular weight $60 \mathrm{Da}$ ) were purchased from Sigma Chemical Company (St Louis, MO). PSA (molecular weight $34 \mathrm{kDa}$ ) was purchased from Enzo Life Sciences (Farmingdale, NY). Osteopontin (molecular weight $33 \mathrm{kDa}$ ) was purchased from Acris Antibodies (San Diego, CA). The urea assay kit was purchased from BioVision (Mountain View, CA). A PSA BioAssay ${ }^{\mathrm{TM}}$ enzyme-linked immunosorbent assay kit was purchased from United States Biological (Swampscott, MA). An osteopontin enzyme immunometric assay kit was purchased from Assay Designs (Ann Arbor, MI). Deionized water (resistivity $\geq 18 \mathrm{M} \Omega \mathrm{cm}$ ) was used for preparation of all solutions,. The deionized water was purified by a Millipore Milli-Q UFplus System (Bedford, MA).

\section{Equipment}

For all in vitro extraction experiments, a diffusion cell was used and was the same as previously described (Figure 1). ${ }^{20}$ Homemade electroporation and reverse iontophoresis devices were used to apply the necessary electric pulse and current, respectively, to the diffusion cell via silver-silver chloride $(\mathrm{Ag} / \mathrm{AgCl})$ electrodes. The electroporation device has an accuracy of $\pm 0.01 \mathrm{msec}$ on timing and $\pm 0.1 \mathrm{~V}$ on delivering voltage, whereas the reverse iontophoresis device has an accuracy of $\pm 0.01 \mathrm{msec}$ on timing and $\pm 0.01 \mu \mathrm{A}$ on delivering current. For $\mathrm{Ag} / \mathrm{AgCl}$ electrode fabrication, a silver wire (0.1 cm diameter, $2.5 \mathrm{~cm}$ length, 99.99\% pure; Aldrich Chemical Company Inc, Milwaukee, WI) was chloridized by immersion in $100 \mathrm{mM} \mathrm{HCl}$ solution, with the platinum electrode as the cathode for 1.5 hours at an applied constant current of $0.314 \mathrm{~mA}$. All colorimetric analysis was performed using a Bio-Rad 680 microplate reader (Bio-Rad, Hemel Hempstead, UK). An impedance analyzer (Precision Impedance Analyzer WK6420C, Wayne Kerr Electronics Ltd, UK) was used for skin impedance measurements.

\section{In vitro studies}

All in vitro experiments were performed using a diffusion cell, in two halves, comprising two upper electrode chambers and a lower chamber. Porcine ear skin $(0.25 \mathrm{~mm})$, obtained from Padgett Dermatome (Integra LifeSciences Corporation, 


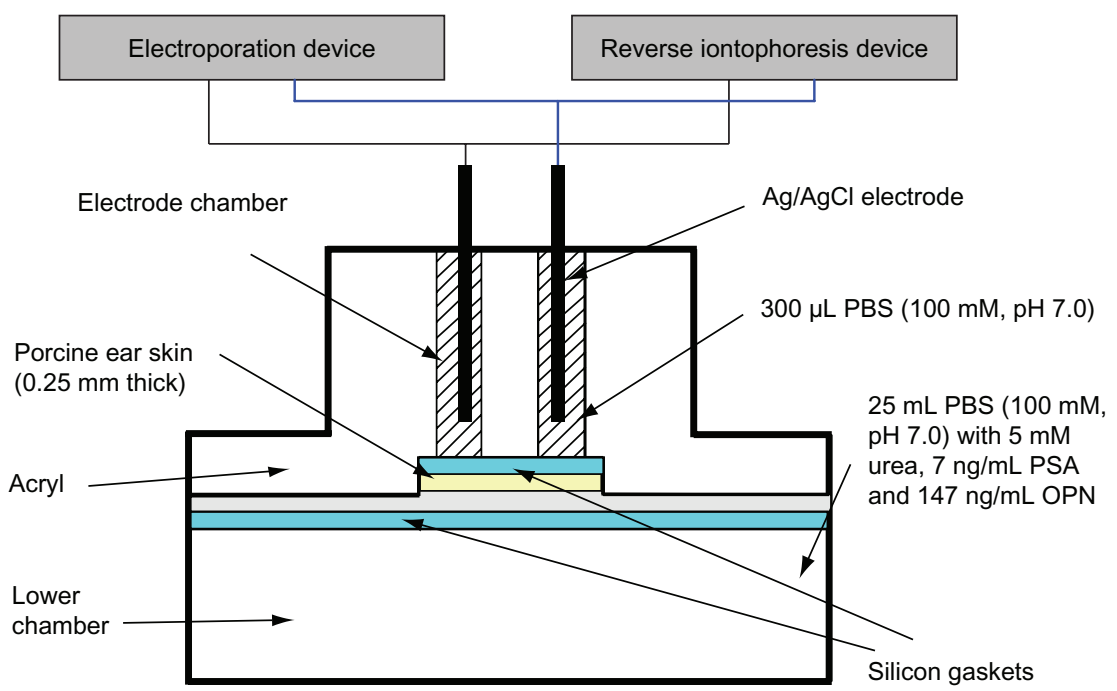

Figure I Diffusion cell for in vitro extraction experiments. The function of the silicon gasket is to prevent leakage of liquid between the electrode chambers and to ensure that current only passes through the skin without any current leakage between electrode chambers.

This figure is modified from Lee et al. ${ }^{21}$

Abbreviations: PBS, phosphate-buffered saline; PSA, prostate-specific antigen; OPN, osteopontin.

Plainsboro, NJ) was used to separate the two upper electrode chambers from the lower chamber. Phosphate-buffered saline (100 mM, pH 7.0) was used to fill the two upper electrode chambers $(300 \mu \mathrm{L}$ each) and the lower chamber (about $25 \mathrm{~mL}$ ). However, the lower chamber also contained $5 \mathrm{mM}$ urea, $7 \mathrm{ng} /$ $\mathrm{mL}$ PSA, and $147 \mathrm{ng} / \mathrm{mL}$ osteopontin. Solution in the lower chamber acts as the interstitial fluid under the skin at normal physiological levels. Each electrode chamber contained a Ag/ $\mathrm{AgCl}$ electrode, with each electrode placed approximately $0.1 \mathrm{~cm}$ above the porcine skin. The surface area of the porcine skin exposed to the electrode in each chamber was $0.2 \mathrm{~cm}^{2}$ and the electrode chambers were $1.1 \mathrm{~cm}$ apart. Different modes of electroporation and reverse iontophoresis (Table 1 and Figure 2) were applied to the diffusion cell via the $\mathrm{Ag} / \mathrm{AgCl}$ electrodes. The entire contents of the electrode chambers were removed at the end of each experiment to determine the amount of urea, PSA, and osteopontin extracted.
Immediately before and after each extraction experiment (see Figure 2), pre-experimental skin impedance $\left(\mathrm{Z}_{\text {before }}\right)$ and post-experimental skin impedance $\left(Z_{\text {after }}\right)$ were measured at $20 \mathrm{~Hz}$ via the $\mathrm{Ag} / \mathrm{AgCl}$ electrodes using an impedance analyzer in order to compute the ratio of pre-experimental to postexperimental skin impedance (ie, $\mathrm{Z}_{\text {before }} / \mathrm{Z}_{\text {after }}$ ). The amplitude of the perturbing wave was limited to $200 \mathrm{mV}$. In all cases, three separate sets of measurements were made in succession in order to check for reliability of measurements. Pictures of the skin surface were also captured by a microscope immediately before and after each extraction experiment, in order to investigate for any changes in the skin surface after the experiment.

\section{Determination of extracted urea, PSA, and osteopontin}

A colorimetric clinical chemistry assay method adapted for use with the Bio-Rad microplate reader was used to quantify

Table I Experimental protocol to show various combinations of electroporation and reverse iontophoresis for in vitro extraction experiments

\begin{tabular}{|c|c|c|c|c|c|}
\hline \multirow{4}{*}{$\begin{array}{l}\text { Urea, PSA, and osteopontin } \\
\text { extraction }\end{array}$} & \multicolumn{5}{|c|}{ Electroporation parameters } \\
\hline & \multicolumn{5}{|c|}{ Pulse width $=1 \mathrm{msec}$} \\
\hline & \multicolumn{5}{|c|}{ Number of pulses per second $=10$} \\
\hline & No voltage ${ }^{b}$ & $74 \mathrm{~V} / \mathrm{cm}$ & $148 \mathrm{~V} / \mathrm{cm}$ & $296 \mathrm{~V} / \mathrm{cm}$ & $592 \mathrm{~V} / \mathrm{cm}$ \\
\hline \multicolumn{6}{|c|}{ Reverse iontophoresis parameters } \\
\hline \multicolumn{6}{|c|}{ Symmetrical biphasic dc $\left(0.3 \mathrm{~mA} / \mathrm{cm}^{2}\right)$} \\
\hline \multicolumn{6}{|l|}{ No current ${ }^{\mathrm{a}}$} \\
\hline \multicolumn{6}{|l|}{30 second phase duration } \\
\hline I80 second phase duration & & & & & \\
\hline
\end{tabular}

Notes: a No current: 0 second phase duration acting as control group; ${ }^{b}$ no voltage: $0 \mathrm{~V} / \mathrm{cm}$ acting as control group. Abbreviation: PSA, prostate-specific antigen. 


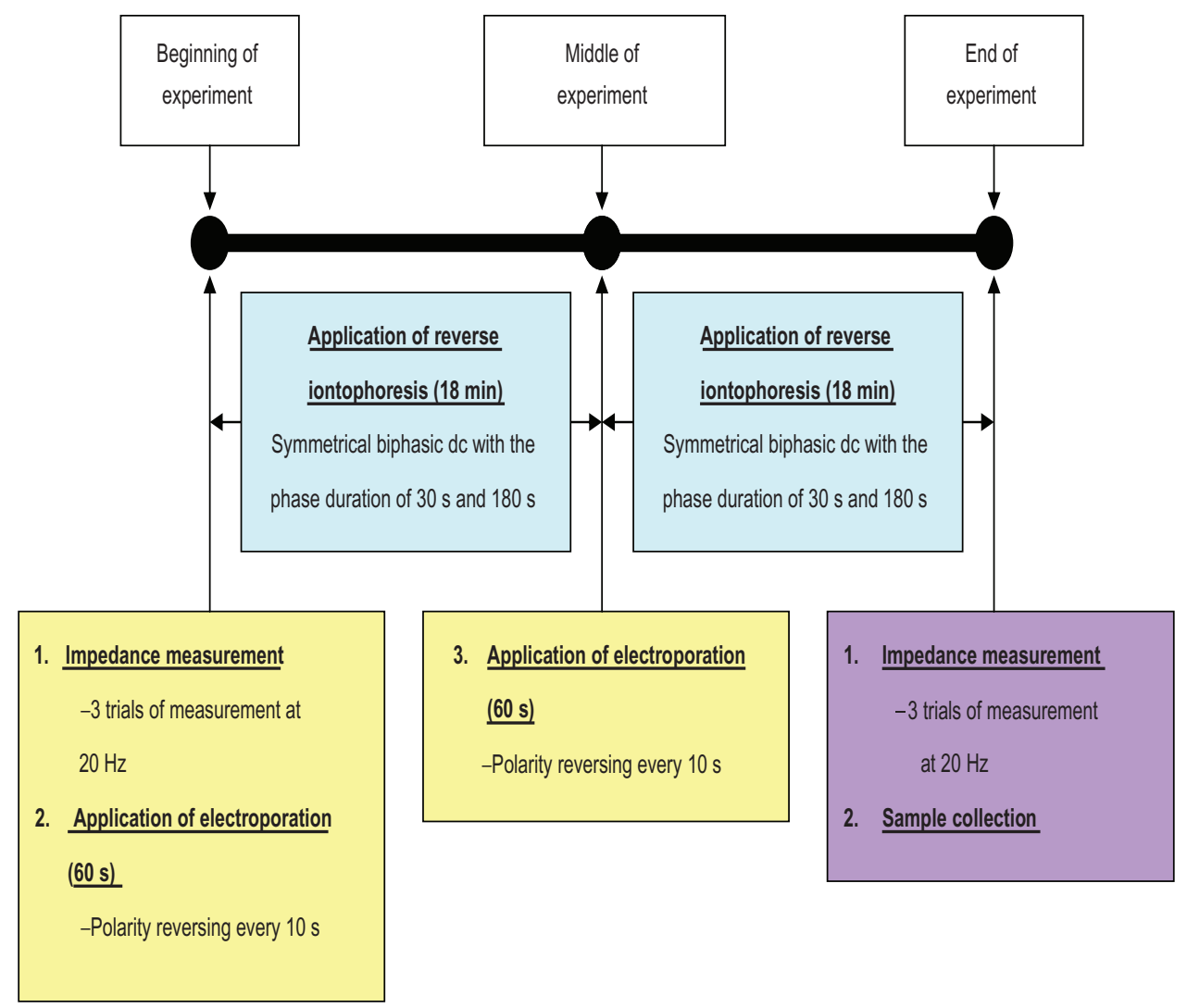

Figure 2 Experimental protocol showing the time sequence for skin impedance measurements, application of electroporation and reverse iontophoresis, and sample collection. Note: Each in vitro extraction experiment took 38 minutes.

Abbreviations: min, minutes; s, seconds.

the amount of urea, PSA, and osteopontin, using their corresponding assay kits and assay procedures mentioned in the user manuals. For the urea assay, $50 \mu \mathrm{L}$ of the extracted solution or standard solution was used. Incubation was at $37^{\circ} \mathrm{C}$ for 60 minutes in a dark environment, and the optical density of the mixed solutions (standard/extracted solutions and quantifying reagents) was read at $570 \mathrm{~nm}$. For the PSA assay, $50 \mu \mathrm{L}$ of the extracted solution or standard solution was used. Incubation was at $37^{\circ} \mathrm{C}$ for 30 minutes, and the optical density of the mixed solutions was read at $450 \mathrm{~nm}$. For the osteopontin assay, $100 \mu \mathrm{L}$ of the extracted solution or standard solution was used. Incubation was at $25^{\circ} \mathrm{C}$ for 30 minutes on a plate shaker at about $500 \mathrm{rpm}$. The optical density of the mixed solutions was read at $405 \mathrm{~nm}$. Excellent linear relationships between urea $\left(r^{2}>0.95\right)$, PSA $\left(r^{2}>0.92\right)$, and osteopontin $\left(r^{2}>0.90\right)$ concentrations and their relative absorbance were found. This allowed the urea, PSA, and osteopontin concentrations to be calculated simply by their linear regressions.

\section{Statistical analysis}

One-way analysis of variance was used to determine whether there were significant differences between reverse iontophoresis parameters within an electroporation parameter, and between electroporation parameters within a reverse iontophoresis parameter for urea, PSA, and osteopontin extraction. One-way analysis of variance was also used to determine whether there were significant differences between reverse iontophoresis parameters and the electroporation parameter for the test parameters of $Z_{\text {before }} / Z_{\text {after }}$. Post hoc comparisons were made with the least significant difference test. All statistical analyses were carried out using SPSS software (SPSS Inc, Chicago, IL), with the level of statistical significance set at 0.05 .

\section{Results}

Our in vitro studies of transdermal and noninvasive extraction of urea, PSA, and osteopontin by different combinations of electroporation and reverse iontophoresis are summarized in Figures 3-5. From Figure 3, it can be seen that reverse iontophoresis, with or without combined use of electroporation, can generally facilitate more transdermal urea extraction as compared with the controls (no reverse iontophoresis and electroporation applications, ie, diffusion only) as well as the groups receiving electroporation alone. On the other hand, 


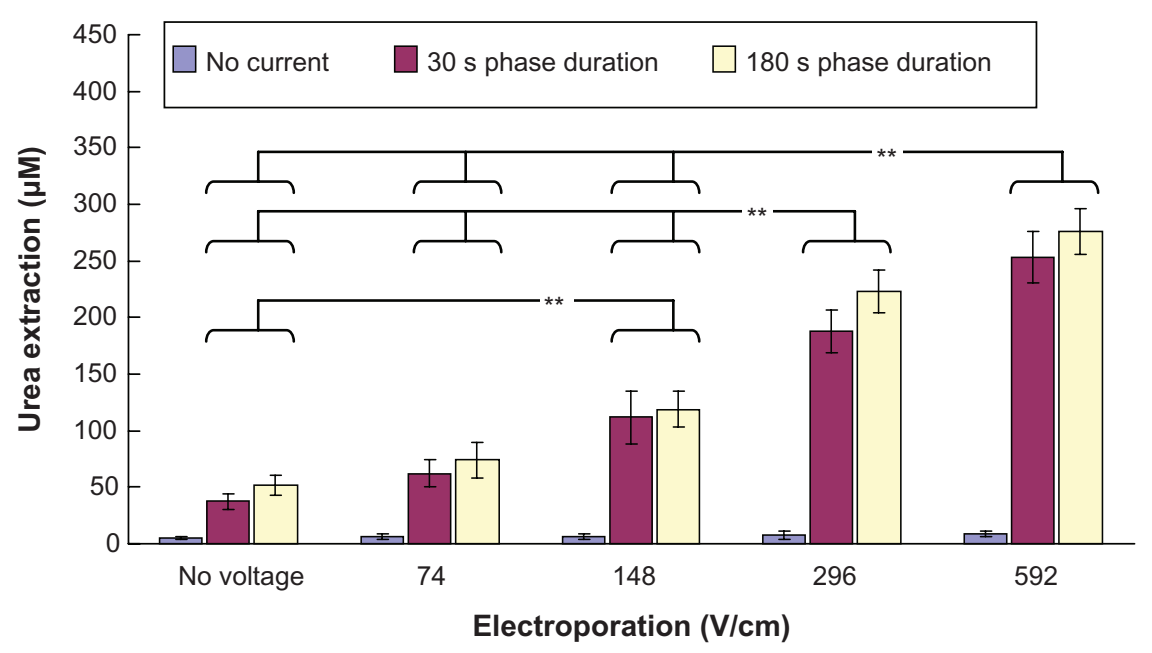

Figure 3 In vitro studies ( $\mathrm{n}=3$ for each bar) of the transdermal and noninvasive extraction of urea by electroporation, reverse iontophoresis, and a combination of these methods. For the electroporation setting, they all have the same pulse width of I msec and number of pulses per second of I0. The legend shows the reverse iontophoresis setting, which is a symmetrical biphasic dc with a current density of $0.3 \mathrm{~mA} / \mathrm{cm}^{2}$.

Notes: Results are expressed as the mean \pm standard deviation. $* * P<0.001$.

urea extraction by reverse iontophoresis, having a phase duration of 180 seconds, was generally observed to be higher than that of 30 seconds, but no significant difference was found. Further, in the same reverse iontophoresis setting, it was found that urea extraction generally could be enhanced by an increase in the electrical field strength of electroporation. Moreover, in the same reverse iontophoresis setting (phase duration 30 seconds or 180 seconds), urea extraction by electroporation at $592 \mathrm{~V} / \mathrm{cm}$ was found to be significantly higher $(P<0.001)$ than that by electroporation at no voltage, and 74 and $148 \mathrm{~V} / \mathrm{cm}$. Also, electroporation at $296 \mathrm{~V} / \mathrm{cm}$ was found to enhance $(P<0.001)$ urea extraction significantly more than electroporation at no voltage, and 74 and $148 \mathrm{~V} /$ $\mathrm{cm}$. Electroporation at $148 \mathrm{~V} / \mathrm{cm}$ was also found to increase urea extraction significantly $(P<0.001)$ compared with electroporation at no voltage.

Figure 4 shows transdermal extraction of PSA by different combinations of electroporation and reverse iontophoresis. It was observed that diffusion (no reverse iontophoresis or electroporation) and reverse iontophoresis

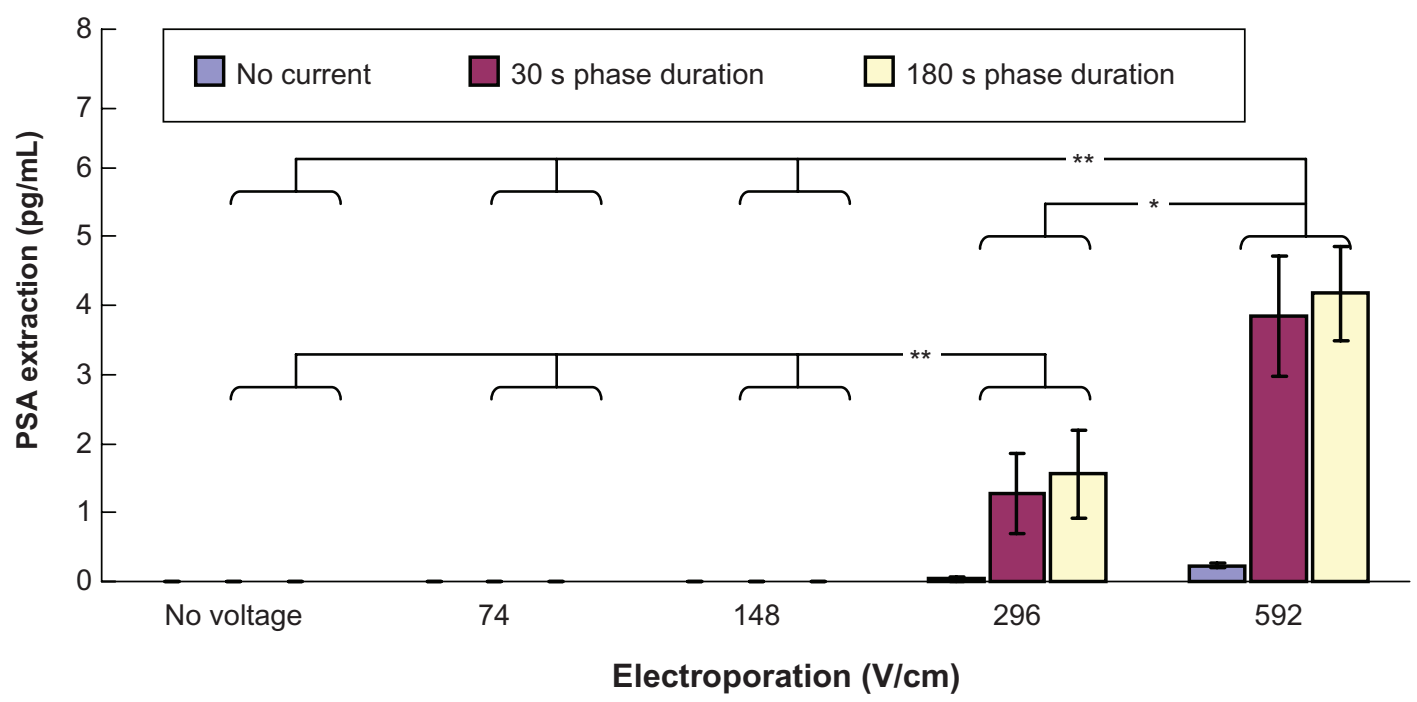

Figure 4 In vitro studies ( $n=3$ for each bar) of transdermal and noninvasive extraction of PSA by various electroporation, reverse iontophoresis, and a combination of the two methods. For the electroporation setting, they all have the same pulse width of I msec and number of pulses per second of 10 . The legend shows the reverse iontophoresis setting, which is a symmetrical biphasic dc with a current density of $0.3 \mathrm{~mA} / \mathrm{cm}^{2}$.

Notes: Results are expressed as mean \pm standard deviation. $* * P<0.001$, $* P<0.05$.

Abbreviation: PSA, prostate-specific antigen. 


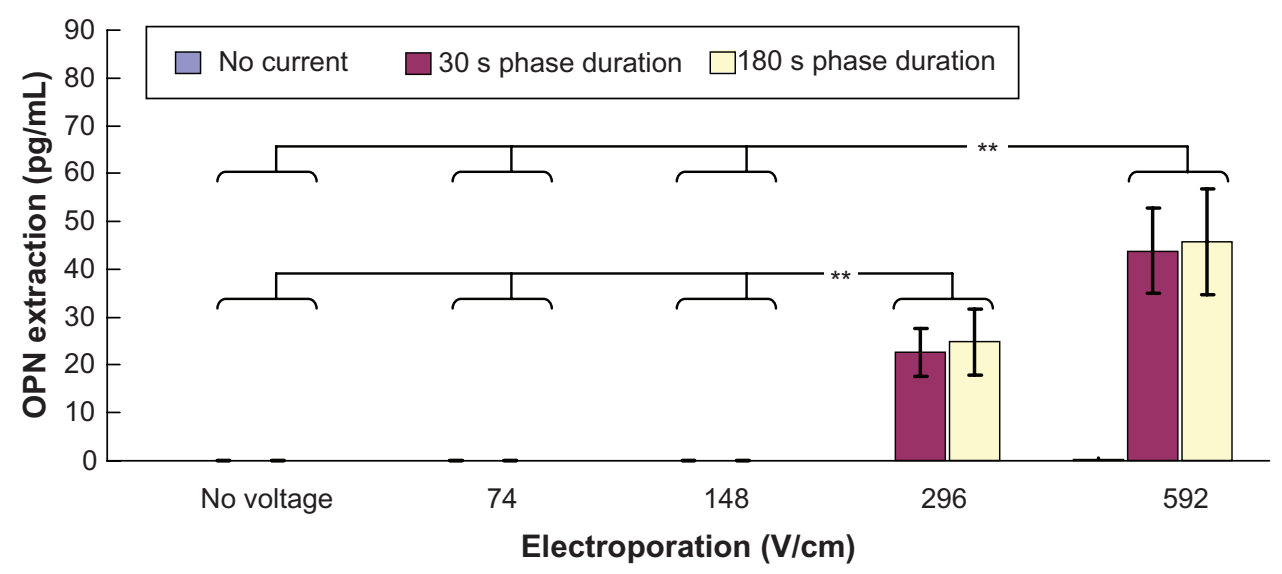

Figure 5 In vitro studies ( $n=3$ for each bar) of transdermal and noninvasive extraction of osteopontin by various electroporation, reverse iontophoresis, and a combination of the two methods. For the electroporation setting, they all have the same pulse width of I msec and the number of pulses per second of I0. The legend shows the reverse iontophoresis setting, which is a symmetrical biphasic dc with a current density of $0.3 \mathrm{~mA} / \mathrm{cm}^{2}$.

Notes: Results are expressed as the mean \pm standard deviation. $* * P<0.001$.

Abbreviation: OPN, osteopontin.

alone cannot facilitate transdermal PSA extraction. On the other hand, electroporation alone with high voltage densities ( 296 and $592 \mathrm{~V} / \mathrm{cm}$ ) was found to induce extremely small PSA transdermal extraction. Importantly, it was found that combining high electroporation (either 296 or $592 \mathrm{~V} / \mathrm{cm}$ ) with reverse iontophoresis (phase duration 30 seconds or 180 seconds) could achieve more PSA extraction than electroporation alone with high voltage densities of 296 and $592 \mathrm{~V} / \mathrm{cm}$. Moreover, with application of reverse iontophoresis and under the same reverse iontophoresis setting (phase duration 30 seconds or 180 seconds), PSA extraction by electroporation at $592 \mathrm{~V} / \mathrm{cm}$ was found to be significantly higher than that by electroporation at no voltage, and 74 and $148 \mathrm{~V} / \mathrm{cm}(P<0.001)$ and electroporation at $296 \mathrm{~V} / \mathrm{cm}$ $(P<0.05)$. Also, with application of reverse iontophoresis and under the same reverse iontophoresis setting (phase duration 30 seconds or 180 seconds), PSA extraction by electroporation at $296 \mathrm{~V} / \mathrm{cm}$ was found to be significantly $(P<0.001)$ higher than that by electroporation at no voltage, and 74 and $148 \mathrm{~V} / \mathrm{cm}$.

Figure 5 shows transdermal extraction of osteopontin by various combinations of electroporation and reverse iontophoresis. It was observed that diffusion (no reverse iontophoresis or electroporation), electroporation, or reverse iontophoresis alone cannot facilitate osteopontin transdermal extraction. On the other hand, it was found that combining higher electroporation at either 296 or $592 \mathrm{~V} / \mathrm{cm}$ with reverse iontophoresis (phase duration 30 or 180 seconds) can facilitate transdermal extraction of osteopontin. Moreover, with application of reverse iontophoresis, osteopontin extraction by electroporation at no voltage, and 74 and $148 \mathrm{~V} / \mathrm{cm}$ was found to be significantly smaller $(P<0.001$ for all cases $)$ than that by electroporation at 296 and $592 \mathrm{~V} / \mathrm{cm}$.

Figure 6 shows the effect of electroporation and reverse iontophoresis on the ratio of pre-experimental to post-experimental skin impedance. It was observed that the diffusion process (ie, no reverse iontophoresis or electroporation) results in a small reduction of skin impedance while electroporation alone, reverse iontophoresis alone, or combinations of electroporation and reverse iontophoresis cause a huge reduction of skin impedance as compared with the diffusion process. On the other hand, reduction of post-experimental skin impedance caused by reverse iontophoresis having a phase duration of 180 seconds was generally observed to be similar to that of 30 seconds, and no significant difference was found. Further, under the same reverse iontophoresis setting, it was found that the increase of electrical field strength of electroporation could generally further decrease postexperimental skin impedance.

Figure 7 shows the ratio of pre-experimental skin impedance to post-experimental skin impedance at various times after application of electroporation (electric field strength $=592 \mathrm{~V} / \mathrm{cm}$; pulse width $=1 \mathrm{msec}$; number of pulses per second $=10$ ) and reverse iontophoresis (symmetrical biphasic dc; current density $=0.3 \mathrm{~mA} / \mathrm{cm}^{2}$; phase duration $=180$ seconds). No significant changes were found, but a steady decrease in the ratio with time was observed.

Figure 8 shows the pictures of the skin surface before and after each extraction experiment. No significant changes at the skin surface were observed after application of electroporation and reverse iontophoresis. 


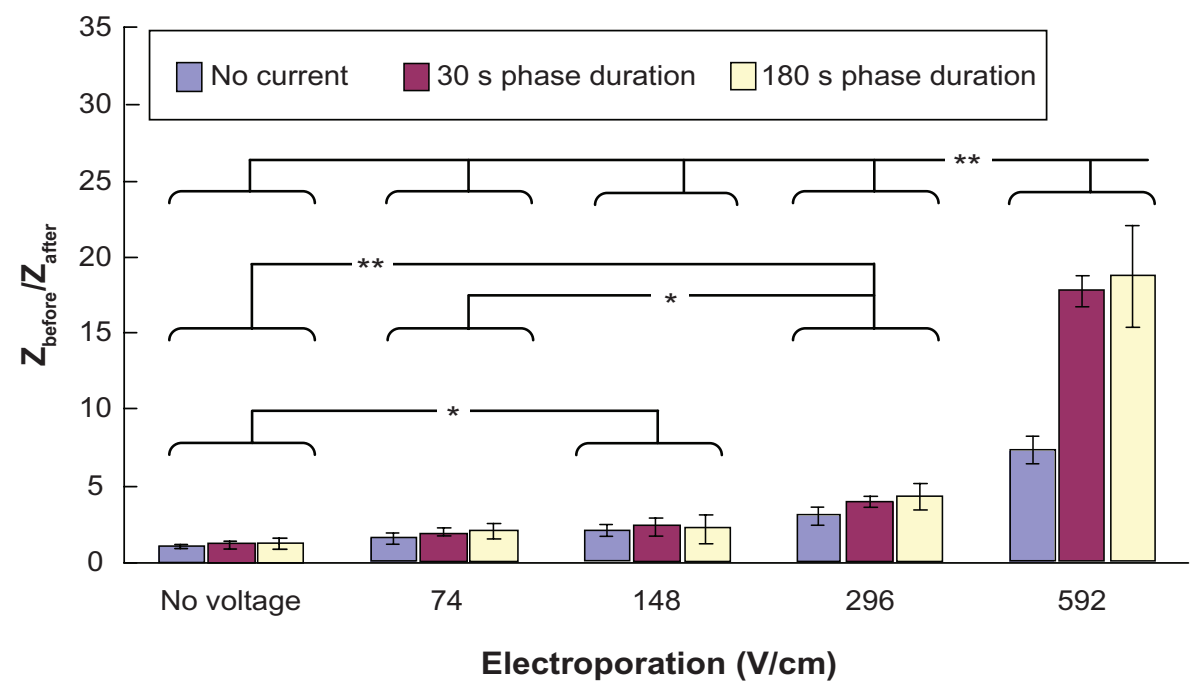

Figure 6 Effect of electroporation and reverse iontophoresis on the ratio of pre-experimental to post-experimental skin impedance (ie, $Z_{\text {before }} / Z_{\text {after }}$ ). All skin impedance measurements were conducted at $20 \mathrm{~Hz}$. For the electroporation setting, they all have the same pulse width of I msec and the number of pulses per second of I 0 . The legend shows the reverse iontophoresis setting, which is a symmetrical biphasic dc with the current density of $0.3 \mathrm{~mA} / \mathrm{cm}^{2}$.

Notes: Results $(n=3)$ are expressed as the mean \pm standard deviation. $* * p<0.001 ; * P<0.05$, respectively.

\section{Discussion}

In this study, all transdermal extraction experiments were conducted using a diffusion cell in order to establish an in vitro model, which has the advantages of simplicity, easy handling, inexpensiveness, and an ability to simulate disease conditions. Although an in vivo model is a good choice for transdermal extraction experiments, it is relatively expensive, and it is quite difficult to create the necessary disease conditions. In this study, a normal physiological in vitro model (5 $\mathrm{mM}$ urea, $7 \mathrm{ng} / \mathrm{mL}$ PSA, and $147 \mathrm{ng} / \mathrm{mL}$ osteopontin) was established in order to simulate a healthy person with normal blood urea, PSA, and osteopontin levels. As shown in Figures 3-5, the results for urea, PSA, and osteopontin extraction, respectively, show that the maximum detected values were much less than the normal physiological values for these biomarkers. This is because all these biomarkers in interstitial fluid, rather than in blood, have been extracted and the concentration of these biomarkers in the interstitial fluid is relatively lower than that in blood. Moreover, the skin provides a barrier to reduce the extraction of these biomarkers further. Our results are in agreement with others who also reported that the maximum detected value of glucose is much less than the normal physiological value in blood. ${ }^{22}$

Urea is a negatively charged molecule with a small molecular weight of approximately $60 \mathrm{Da}$. As expected, reverse iontophoresis alone can enhance transdermal extraction of

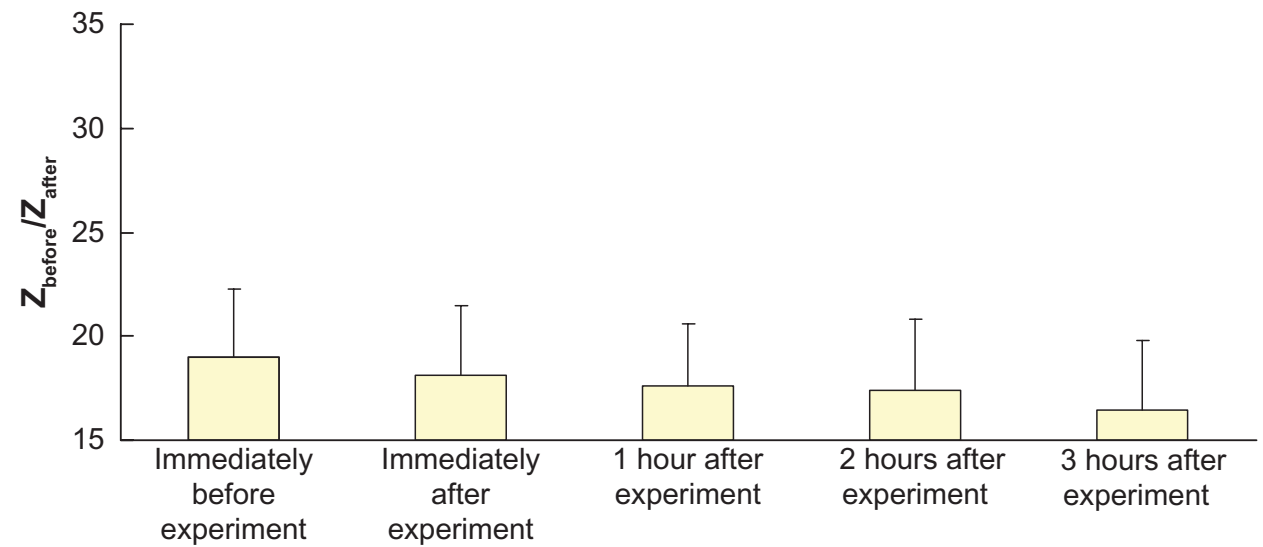

Figure 7 Effect of electroporation (electric field strength $=592 \mathrm{~V} / \mathrm{cm}$; pulse width $=1$ msec; number of pulses per second $=10$ ) and reverse iontophoresis (symmetrical biphasic dc; current density $=0.3 \mathrm{~mA} / \mathrm{cm}^{2}$; phase duration $=180$ seconds) on the ratio of pre-experimental to post-experimental skin impedance at various times after the extraction experiment. 
A

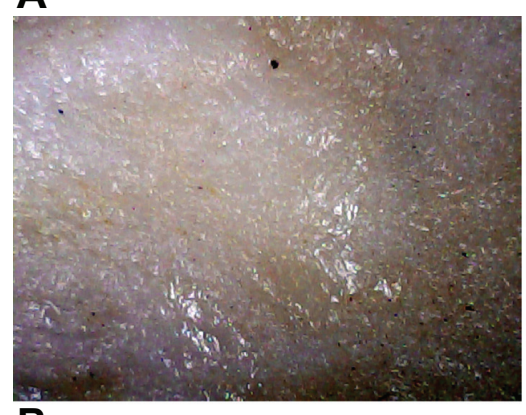

B

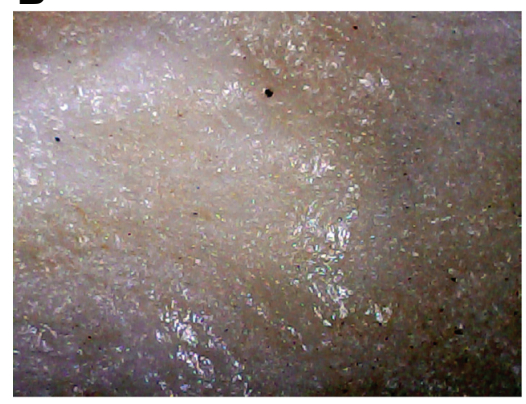

C

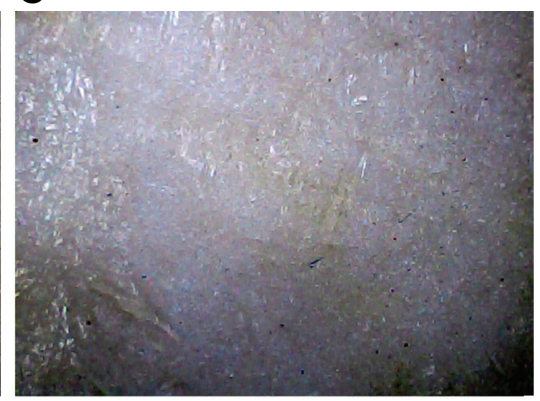

D

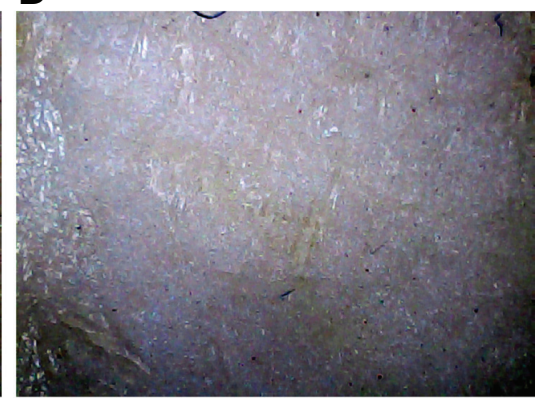

Figure 8 Pictures of the skin surface before and after each extraction experiment. All pictures were captured by a microscope with a magnification of $60 \times$. (A and B) Before and after the extraction experiment, respectively, with application of electroporation $(74 \mathrm{~V} / \mathrm{cm})$ and reverse iontophoresis (phase duration $=180$ seconds). $(\mathbf{C}$ and $\mathbf{D})$ Before and after extraction experiment, respectively, with application of electroporation $(592 \mathrm{~V} / \mathrm{cm}$ ) and reverse iontophoresis (phase duration $=180$ seconds).

urea through the process of electromigration, and further urea extraction can be achieved using reverse iontophoresis together with electroporation (see Figure 3). A possible explanation for the phenomenon observed in Figure 3 is that electroporation can create nanochannels within the skin, ${ }^{23,24}$ and these nanochannels provide a low travelling resistance pathway for urea to pass through. Researchers have shown that a low and long-lasting voltage pulse $(\leq 5 \mathrm{kV} / \mathrm{cm}$, about $500 \mu \mathrm{sec})$ can produce pores with diameters of $10-50 \mu \mathrm{m}^{23}$ and even up to $600 \mu \mathrm{m} .{ }^{24}$ The higher the electrical field strength of the electroporation used, the more/larger the nanochannels that are created, resulting in increased transdermal urea extraction. The findings shown in Figure 6 agree with this hypothesis. After application of electroporation to the skin, a reduction in post-experimental skin impedance is observed, and this may be due to formation of nanochannels. The resulting drop in skin impedance is effectively proportional to the electrical exposure dose (voltage $\times$ total pulse duration). ${ }^{25}$ As shown in Figure 6, the increased electrical field strength of electroporation causes further reduction in post-experimental skin impedance, and this may be due to more/larger nanochannels being formed.

PSA is a negatively charged large molecule with a molecular weight of about $34 \mathrm{kDa}$. Because PSA is a large molecule, it is expected that PSA extraction by reverse iontophoresis is facilitated by electro-osmosis but not by electromigration. It is well known that reverse iontophoresis extraction efficiency facilitated by electroosmosis is much lower than that by electromigration. Hence, as expected, reverse iontophoresis alone cannot facilitate PSA transdermal extraction (see Figure 4) because of its large molecular size. This is because transdermal extraction enhanced by reverse iontophoresis is limited to molecules having a molecular weight of $20 \mathrm{kDa}$ or less, because the main permeable pathway created by reverse iontophoresis is for the skin appendages, such as hair follicles and sweat glands. ${ }^{16}$ As shown in Figure 4, electroporation alone with a high electrical field strength of $296 \mathrm{~V} / \mathrm{cm}$ was found to be able to induce extremely small PSA transdermal extraction. This shows that electroporation at $296 \mathrm{~V} / \mathrm{cm}$ can help to create nanochannels within the skin, ${ }^{23,24}$ which are just large enough (perhaps about $34 \mathrm{kDa}$ ) for passage of PSA molecules. On the other hand, slightly more PSA transdermal extraction can be achieved by electroporation at $592 \mathrm{~V} / \mathrm{cm}$ alone as compared with electroporation at $296 \mathrm{~V} / \mathrm{cm}$ alone. This shows that electroporation at $592 \mathrm{~V} / \mathrm{cm}$ is able to create more/larger nanochannels as compared with electroporation at $296 \mathrm{~V} / \mathrm{cm}$. It is well known that the combination of electroporation and reverse iontophoresis has additional benefits on molecule extraction across the skin. It was observed that electroporation at 296 or $592 \mathrm{~V} / \mathrm{cm}$ together with application of reverse iontophoresis facilitates 
considerable PSA extraction. A possible explanation for the phenomenon observed in Figure 4 is that electroporation with higher electrical field strength can cause more/larger nanochannels to be created, and this causes the increase in transdermal PSA extraction. Again, the finding shown in Figure 6 agrees with this hypothesis and the explanation is the same as described above. On the other hand, it was observed that electroporation with a higher electrical field strength can enhance skin permeability of molecules with a molecular weight of $34 \mathrm{kDa}$. Our finding is in agreement with those reported by other researchers, who suggest that electroporation can increase skin permeability of molecules with a molecular weight of at least $40 \mathrm{kDa} \cdot{ }^{18,19}$ Osteopontin is a large molecule carrying a negative charge. It has a molecular weight of about $33 \mathrm{kDa}$. Given that osteopontin has a large molecular size, it is expected that osteopontin extraction by reverse iontophoresis is enhanced by electroosmosis rather than electromigration. Reverse iontophoresis extraction efficiency facilitated by electro-osmosis is much lower than that by electromigration. Therefore, owing to its large molecular size and low extraction efficiency, reverse iontophoresis alone cannot facilitate transdermal osteopontin extraction (see Figure 4) and this is within our expectations. Again, it was observed that electroporation alone with a high electrical field strength $(592 \mathrm{~V} / \mathrm{cm})$ could induce small transdermal osteopontin extraction. With the aid of reverse iontophoresis, electroporation at 296 or $592 \mathrm{~V} / \mathrm{cm}$ was also found to facilitate considerable osteopontin extraction. The explanation is the same as described above.

Based on the findings shown in Figures 3-5, it is possible to have simultaneous transdermal extraction of urea, PSA, and osteopontin by reverse iontophoresis together with electroporation, provided that electroporation has a high electrical field strength of at least $296 \mathrm{~V} / \mathrm{cm}$.

Self-normalization concept has been employed in the test parameter of $Z_{\text {before }} / Z_{\text {after }}$ in order to avoid the large pig-to-pig differences in skin. $Z_{\text {before }} / Z_{\text {after }}$ can eliminate the effect of pig-to-pig variation during data analysis and therefore allows the skin impedance to be studied without the nonuniformities introduced by pig-to-pig variation. For the test parameter of $Z_{\text {before }} / Z_{\text {after }}$, if its value is greater than 1 , it means that the post-experimental skin impedance is smaller than the pre-experimental skin impedance. As shown in Figure 6 , it was found that all the values of $Z_{\text {before }} / Z_{\text {after }}$ are greater than 1 . This means that the value of skin impedance decreases after the application of reverse iontophoresis, electroporation, or a combination of the two techniques. During reverse iontophoresis, skin may be polarized, and ion (eg, $\mathrm{H}^{+}$and $\mathrm{OH}^{-}$) accumulation occurs inside the skin and this may be the reason for the reduction of skin impedance after application of reverse iontophoresis. During electroporation, nanochannels are formed on the skin. ${ }^{23,24}$ Therefore, the resistance of the skin barrier for the impedance current to flow decreases, and the impedance value decreases consequently. As shown in Figure 6, the increase of electric field strength of the electroporation results in more reduction of the postexperimental skin impedance. A possible explanation for this phenomenon is that more/larger nanochannels are formed if electroporation with a higher electrical field strength is used. Our results agree with those of others showing that the resulting drop in skin impedance is effectively proportional to the electrical exposure dose (voltage $\times$ total pulse duration). ${ }^{25}$

As shown in Figure 7, although no significant changes were observed in the values of $Z_{\text {before }} / Z_{\text {after }}$ with time after application of electroporation (electric field strength $=592 \mathrm{~V} / \mathrm{cm}$; pulse width $=1 \mathrm{msec}$; number of pulses per second $=10$ ) and reverse iontophoresis (symmetrical biphasic dc; current density $=0.3 \mathrm{~mA} / \mathrm{cm}^{2}$; phase duration $=180$ seconds , an extremely small decrease in the value of $Z_{\text {before }} / Z_{\text {after }}$ was detected with time. This meant that post-experimental skin impedance gradually increased with time. The increase in post-experimental skin impedance may be due to recovery of the skin, leading to reduction of the amount and size of the nanopore.

Finally, although PSA and osteopontin can be transdermally extracted, electroporation with a high electrical field strength of at least $296 \mathrm{~V} / \mathrm{cm}$ should be used. Therefore, it is not clinically feasible for safety reasons and induction of pain sensation and involuntary muscle contractions.

\section{Conclusion}

A new method for noninvasive transdermal extraction of PSA and osteopontin by reverse iontophoresis together with electroporation has been demonstrated. Reverse iontophoresis alone cannot facilitate transdermal extraction of PSA and osteopontin, while application of electroporation has a further enhancement effect on molecule extraction. The higher the electrical field strength of electroporation, the higher the enhancement effect on molecule extraction. Finally, it is possible to have simultaneous transdermal extraction of urea, PSA, and osteopontin by reverse iontophoresis together with electroporation, on the condition that electroporation has a high electrical field strength of at least $296 \mathrm{~V} / \mathrm{cm}$. However, because of the need for electroporation with a high electrical field strength, this method is not clinically feasible. 


\section{Acknowledgments}

This work was supported by a grant (TCVGH-NCNU997901) from the Taichung Veterans General Hospital and National Chi Nan University, Taiwan, Republic of China. This work was also supported by grants (NSC 100-2221-E260-007-, NSC 99-2221-E-260-004-, and NSC 98-2221-E260-024-MY3) from the National Science Council, Taiwan, and was partially supported by a grant (100T1100) from the National Chi Nan University, Taiwan.

\section{Disclosure}

The authors report no conflicts of interest in this work.

\section{References}

1. Zieve D, Miller S. Prostate cancer, 2010. Available from: http://www. nlm.nih.gov/medlineplus/ency/article/000380.htm. Accessed October 16, 2011.

2. Department of Health, Executive Yuan, ROC (Taiwan). Part II. Statistics on Causes of Death, 2005. Available from: http://www.doh.gov.tw/ EN2006/DM/DM2.aspx?now_fod_list_no=9377\&class_no=390\&level_ no=2. Accessed October 16, 2011.

3. Department of Health, Executive Yuan, ROC (Taiwan). Health Statistics in Taiwan, 2009. Available from: http://www.doh.gov.tw/EN2006/ DM/DM2.aspx?now_fod_list_no $=9377 \&$ class_no $=390 \& l e v e 1 \_n o=2$. Accessed October 16, 2011.

4. Department of Health, Executive Yuan, ROC (Taiwan). Part II. Statistics on Causes of Death, 2008. Available from: http://www.doh.gov.tw/ EN2006/DM/DM2.aspx?now_fod_list_no=9377\&class_no=390\&level_ no $=2$. Accessed October 16, 2011.

5. Manne U, Srivastava RG, Srivastava S. Recent advances in biomarkers for cancer diagnosis and treatment. Drug Discov Today. 2005;10: 965-976.

6. Song G, Cai QF, Mao YB, Ming YL, Bao SD, Ouyang GL. Osteopontin promotes ovarian cancer progression and cell survival and increases HIF-1alpha expression through the PI3-K/Akt pathway. Cancer Sci. 2008;99:1901-1907.

7. Ye B, Skates S, Mok SC, et al. Proteomic-based discovery and characterization of glycosylated eosinophil-derived neurotoxin and $\mathrm{COOH}$-terminal osteopontin fragments for ovarian cancer in urine. Clin Cancer Res. 2006;12:432-441.

8. Connolly DJ, Black A, Murray LJ, Gavin A, Keane PF. Population based age-specific reference ranges for PSA. Prostate Cancer Symposium 2007, abstract 12 .
9. Schorge JO, Drake RD, Lee H, et al. Osteopontin as an adjunct to CA125 in detecting recurrent ovarian cancer. Clin Cancer Res. 2004;10: 3474-3478.

10. Degim IT, Ilbasmis S, Dundaroz R, Oguz Y. Reverse iontophoresis: a noninvasive technique for measuring blood urea level. Pediatr Nephrol. 2003;18:1032-1037.

11. Pitzer KR, Desai S, Dunn T, et al. Detection of hypoglycaemia with the GlucoWatch biographer. Diabetes Care. 2001;24:881-885.

12. Mize NK, Buttery M, Daddona P, Morales C, Cormier M. Reverse iontophoresis: monitoring prostaglandin $\mathrm{E} 2$ associated with cutaneous inflammation in vivo. Exp Dermatol. 1997;6:298-302.

13. Merino V, López A, Hochstrasser D, Guy RH. Noninvasive sampling of phenylalanine by reverse iontophoresis. J Control Release. 1999;61: 65-69.

14. Murthy SS, Kiran VS, Mathur SK, Murthy SN. Noninvasive transcutaneous sampling of glucose by electroporation. J Diabetes Sci Technol. 2008;2:250-254.

15. Merino V, Kalia YN, Guy RH. Transdermal therapy and diagnosis by iontophoresis. Trends Biotechnol. 1997;15:288-290.

16. Turner NG, Ferry L, Price M, Cullander C, Guy RH. Iontophoresis of poly-1lysines: the role of molecular weight? Pharm Res. 1997;14: 1322-1331.

17. Jadoul A, Bouwstra J, Preat V. Effects of iontophoresis and electroporation on the stratum corneum. Review of the biophysical studies. Adv Drug Deliv Rev. 1999;35:89-105.

18. Tokudome Y, Sugibayashi K. The synergic effects of various electrolytes and electroporation on the in vitro skin permeation of calcein. J Control Release. 2003;92:93-101.

19. Tokudome Y, Sugibayashi K. The effects of calcium chloride and sodium chloride on the electroporation-mediated skin permeation of fluorescein isothiocyanate (FITC)-dextrans in vitro. Biol Pharm Bull. 2003;26:1508-1510.

20. Ching TS, Connolly P. Simultaneous transdermal extraction of glucose and lactate from human subjects by reverse iontophoresis. Int $J$ Nanomedicine. 2008;3:211-223.

21. Lee CK, Ching CT, Sun TP, et al. Non-invasive and transdermal measurement of blood uric acid level in human by electroporation and reverse iontophoresis. Int J Nanomedicine. 2010;5:991-997.

22. Ching CTS, Buisson Y, Connolly P. The effect of pulsed bipolar de on the simultaneous extraction of glucose and lactate by reverse iontophoresis. Sensor Actuat B Chem. 2008;129:504-509.

23. Gowrishankar TR, Pliquett U, Weaver JC. Changes in skin structure and electrical properties following high voltage exposure. Ann NY Acad Sci. 1999;888:183-194.

24. Gowrishankar TR, Herndon TO, Vaughan TE, Weaver JC. Spatially constrained localized transport regions due to skin electroporation. J Control Release. 1999;60:101-110.

25. Gallo SA, Sen A, Hensen ML, Hui SW. Time-dependent ultrastructural changes to porcine stratum corneum following an electric pulse. Biophys $J$. 1999;76:2824-2832.
International Journal of Nanomedicine

\section{Publish your work in this journal}

The International Journal of Nanomedicine is an international, peerreviewed journal focusing on the application of nanotechnology in diagnostics, therapeutics, and drug delivery systems throughout the biomedical field. This journal is indexed on PubMed Central, MedLine, CAS, SciSearch ${ }^{\circledR}$, Current Contents ${ }^{\circledR} /$ Clinical Medicine,

\section{Dovepress}

Journal Citation Reports/Science Edition, EMBase, Scopus and the Elsevier Bibliographic databases. The manuscript management system is completely online and includes a very quick and fair peer-review system, which is all easy to use. Visit http://www.dovepress.com/ testimonials.php to read real quotes from published authors. 